\title{
Do online reviews diminish physician authority? The case of cosmetic surgery in the U.S.
}

\author{
Abstract \\ This article analyzes the substance and perception of online physician reviews, which are \\ qualitative and quantitative assessments of physicians written and shared by patients, in the case \\ of U.S. cosmetic surgery. Like other cash-pay medical specialties, cosmetic surgery is elective \\ and paid for largely out of pocket, with patients having latitude in their choice of surgeon. \\ Drawing on qualitative data from interviews, observations of an American Society of Aesthetic \\ Plastic Surgery professional meeting, and online reviews from the platforms RealSelf and Yelp, I \\ identify two interdependent contributors to physician authority: reputation and expertise. I argue \\ that surgeons see reviews overwhelmingly as a threat to their reputation, even as actual review \\ content often positively reinforces physician expertise and enhances physician reputation. I show \\ that most online reviews linked to interview participants are positive, according considerable \\ deference to surgeons. Reviews add patients' embodied and consumer expertise as a \\ circumscribed supplement to surgeons' technical expertise. Moreover, reviews change the \\ doctor-patient relationship by putting it on display for a larger audience of prospective patients, \\ enabling patients and review platforms to affect physician reputation. Surgeons report changing \\ how they practice to establish and maintain their reputations. This research demonstrates how \\ physician authority in medical consumerist contexts is a product of reputation as well as \\ expertise. Consumerism changes the doctor-patient relationship and makes surgeons feel \\ diminished authority by dint of their reputational vulnerability to online reviews.
}

Keywords: U.S.; cosmetic surgery; internet; medical consumerism; authority; expertise; reputation; doctor-patient relationship 
As online reviews expand in the medical arena in the U.S. (López et al., 2012; Mueller et al., 2013), cosmetic surgery is a good case for examining their effects on the doctor-patient relationship. Free from the mandate of a physician referral and other constraints normally imposed by insurance, cosmetic surgery is notable for its pronounced medical consumerism. Surgeons compete to attract patients through word-of-mouth referrals and marketing amidst recurring professional debates about the appropriateness of advertising cosmetic procedures (Sullivan, 2001). This study investigates whether online reviews of physicians by patients diminish physician authority. I find that reviews can potentially diminish physician authority, changing the doctor-patient relationship. However, I also show that in practice this effect is minor, and that many cosmetic surgeons interpret reviews as a greater threat to their authority than is actually the case.

As with other physicians, the authority of cosmetic surgeons is rooted in patients' dependence on physicians' specialized knowledge and training (Starr 1982) — their expertisebut also on physician reputation. Based on an analysis of reviews coupled with observation of an American Society of Aesthetic Plastic Surgery meeting and interviews with surgeons, I argue that reviews shift the balance of authority in the doctor-patient relationship by providing patients with a means to damage physician reputation. Reviews by patients asserted their lay expertise as consumers, reduced information asymmetries between patients and surgeons, and evaluated surgeons. Even as review content mostly reinforced and complemented physician expertise, surgeons perceived reviews as introducing third-parties (i.e., review platforms and prospective patients) into the doctor-patient relationship, and feared that negative reviews would scare away prospective patients. 
In contrast to health information websites that have previously been studied (Adams, 2014), some review platforms in the U.S. exist largely outside conventional institutional channels of insurance companies, hospitals, or the state. Without such alliances or direct financial ties to medical industries, these platforms offer patients leverage on their providers in the unequal doctor-patient relationship. However, reviews make cosmetic surgeons only superficially accountable to patients on the aesthetic outcomes as well as the service aspects of their practice: I show how surgeons adjusted their practices to avoid reputational harm and maintain their authority.

\section{Online Evaluation: Authority, Expertise, and Reputation}

\section{Authority, Expertise and Reputation in Medicine}

In analyzing how cosmetic surgeons are evaluated by patients, I consider expertise and reputation as contributors to physician authority. Starr $(1982,9)$ describes authority in medicine as "the possession of some status, quality, or claim that compels trust or obedience." Building on Weber, Starr sees physician authority as rooted in the perception of the legitimacy of the doctor's instructions (which derives from their training, credentialing and expertise) and the dependence of patients on physicians. Authority is a power relation based on differential knowledge, with the doctor retaining power due to her "superior competence" (Starr, 1982, 11). Cosmetic surgeons are medical practitioners vested with authority and credentialed by the American Medical Association and state licensing boards. As cues alongside established signals of expertise such as credentials, reviews give patients some power to shape the perception of the legitimacy of a physician and reduce the dependence of patients on any one physician.

Cosmetic surgeons are experts in the sense that they are "sponsored intellectual[s]" with the weight of institutions supporting their claims to knowledge and ability (Fine and Xu, 2012, 
153; Eyal, 2013). This expertise is not solely the province of the credentialed expert but rather as "a network linking together agents, devices, concepts, and institutional and spatial arrangements" (Eyal, 2003, 863), including medical schools, professional organizations, and mastery of special equipment in the case of cosmetic surgery. Patients are also part of the web of expertise, possessing a lay, embodied expertise rooted in their personal experiences in selecting a surgeon, undergoing a procedure, and recovering from surgery (Prior, 2003; Barker, 2008). "Expert" patients contribute information about their health experiences online largely for the benefit of other patients (Lupton, 2014; Adams, 2011), but their assertion of their expertise has been met with some skepticism by doctors (Fox, 2005; Broom, 2005).

Though patients have a form of expertise, they often have limited ability to evaluate the technical expertise of physicians. Patients rely instead on "the stature of the expert" in selecting a surgeon (Fine and $\mathrm{Xu}, 2012,154)$. A surgeon's stature, or reputation, is a function of their public persona and service orientation as well as their technical skill. Reputation is "a socially recognized persona" (Fine and Xu, 2012, 177) or, for our purposes, collective representation of ability and competence. Patients shape their surgeons' reputations by writing reviews and wordof-mouth referrals. Surgeons may try to enhance their reputations through branding. Due to medical privacy laws and the anonymity of online review platforms, patients can anonymously enhance or damage surgeons' reputations by writing reviews reflecting their satisfaction with surgeons' actions.

\section{Online health information and lay expertise}

Recent research describes the development and sharing of patients' lay expertise through the internet. This literature investigates patients' strategies for gathering information, the communities that they form, and the knowledge claims they make based on their health 
experiences (Barker, 2008; Foster, 2016). Lay expertise in this case stems from experience as an informed patient undergoing treatment and experience as a consumer purchasing a medical service (Epstein, 1995; Broom, 2005). Cosmetic surgery reviewers are not only patients and consumers but can also inhabit a third role as producers of online health information (Lupton, 2014). Patients exhibit expertise as informed or reflexive consumers, participating in a larger project of monitoring health professions even as they compare notes on procedures that offer the best value for money (Henwood et al., 2003; Lupton, 1997; Adams, 2014). By influencing surgeons' reputations online, patients can assert themselves without having formal authority over physicians and/or financial resources.

Nevertheless, research suggests that the advent of the internet has not decisively given patients the upper hand. Information asymmetries and power differentials between physicians and patients persist (Conrad et al., 2016). Health information websites have generally re-affirmed conventional medical expertise, even as doctors regard their advent with mistrust (Song et al., 2012; Chiu, 2011). Moreover, in contexts where healthcare is subsidized or provided by the government, writing reviews can be cast as a civic duty (Adams, 2014). Health information websites depend on patients to contribute for free, potentially exploiting patients' efforts (Lupton, 2014). While health information websites position themselves as leveling information asymmetries, scholars suggest that most such websites ally themselves with existing medical institutions, ultimately upholding physician authority (Adams, 2011).

\section{The authority of online reviews and their reputational effects}

For most online health information websites, third-party companies provide platforms for users to share information with one another. Companies guarantee the authenticity of users but not the validity or accuracy of the information they host (Jeacle and Carter, 2011; Orlikowski 
and Scott, 2014). Review platforms supplant expert, impartial, third-party critics such as ranking organizations or travel agencies (Lamont, 2012). Rather than offering objective evaluations of quality (De Langhe et al., 2015), reviews supply patients'subjective evaluations, shaping physicians' reputation without necessarily challenging physician expertise. These reviews derive their authority from volume, with "unregulated contributions by anonymous consumers trump[ing] the legitimized authority of experts and critics" (Orlikowski and Scott, 2014, p.869).

Both the quantitative and qualitative aspects of reviews contribute to their authority. Authority can stem from abstract, complex numerical ratings, averaged and standardized to provide an ostensibly objective approach to trust and evaluation, as shown by Espeland and Sauder (2016) in the case of academic rankings and Porter (1995) in government cost-benefit analysis. But the qualitative, personal, embodied-based narrative comments are also authoritative, even when they contradict quantitative ratings (Orlikowski and Scott, 2014). In evaluating surgeons and giving advice to prospective patients, reviewers subjectively provide "authoritative opinion... which has its origins in the 'authenticity' of the opinions offered" (Jeacle and Carter, 2011). Thanks to a technical tool and standardized commercial product, reviews allow the introduction of others into the doctor-patient relationship.

\section{Medical consumerism and cosmetic surgery}

While cosmetic surgery is unique in the extent of its consumerism, medical consumerism is an increasingly important feature of modern medicine according to theories of biomedicalization (Clarke et al. 2003). Cosmetic surgery consists of invasive and elective aesthetic procedures, such as breast augmentation, requiring a high level of surgical skill/expertise. Without standardized diagnoses or insurance codes, cosmetic surgeons have considerable autonomy in determining medical indications and treatments, harking back to an 
earlier era of strong professional autonomy (Starr, 1982). Indeed, cosmetic surgery lacks the institutional oversight that governs much of modern medicine: surgeons may operate in standalone clinics, circumventing hospital rules, and expert intermediaries claiming to assess quality have never been very successful in this field (Sullivan, 2001). The aim of cosmetic surgery is ultimately subjective; surgeons operate to alleviate the suffering patients experience from a mismatch between their external appearance and internal personality (Haiken, 1997), making patient satisfaction and good rapport between patients and surgeons crucial determinants of success. Medical practitioners from different specializations have competed for years to cater to cosmetic patients (Sullivan, 2001). After the lifting of American Medical Association bans on solicitation of patients in the 1970s, plastic surgery professional associations reversed course and eventually became enthusiastic proponents of marketing. Advertisements helped patients distinguish between a core of board-certified plastic surgeons and otolaryngologists and non-core physicians. The former have undergone years of specialized surgical training and typically charge the highest prices, whereas the latter offer cheaper, ostensibly lower quality services.

This study builds on the existing literature on physician authority and the doctor-patient relationship by considering the effects of reviews on physician authority through mediating components of reputation and expertise. Research has shown both physicians' general wariness of reviews (Patel et al., 2015; Mueller et al., 2013) and the reflexive, thoughtful potential of reviews (Adams, 2011). This article analyzes reviews and surgeons' reactions to them together, showing how even surgeons who receive positive reviews fear their potential threat to their reputations. In what follows, I describe the study's methods; present an analysis of the content of reviews; discuss cosmetic surgeons' impressions of them; and explore possible consequences of the proliferation of reviews on cosmetic surgery practice. 


\section{Data and Methods}

This study employed qualitative data from interviews with cosmetic surgeons and two online review websites. I triangulated findings from these sources with articles published in the Aesthetic Surgery Journal and ethnographic observation from a recent American Society of Aesthetic Plastic Surgery (ASAPS) annual meeting (see supplementary data for more information). Reviews were collected after interviews with cosmetic surgeons were completed. Research protocols were approved by the Northwestern University Institutional Review Board. All respondent names are pseudonyms.

Interviews.

From May to November 2015, I conducted semi-structured interviews with 42 boardcertified plastic and facial plastic surgeons in New York City $(n=11)$, Los Angeles $(n=12)$, Chicago $(n=16)$ and Houston (n=3). In this study, "cosmetic surgeon" denoted board-certified surgeons who conducted cosmetic procedures paid for by patients out-of-pocket. Participants were recruited by email and letter from the rosters of ASAPS, the American Society of Plastic Surgeons, and the American Academy of Facial Plastic and Reconstructive Surgery to participate in a study about cosmetic surgery and expertise. Once they responded, they were informed of the risks and benefits of study participation and provided consent to participate. Conducted in person in surgeons' offices, interviews lasted between forty-five minutes and one hour and were recorded and transcribed verbatim. Thirty respondents were in private solo practice; the rest were based in group clinics, academic centers, and a public hospital. Nine interviewees identified as women and ten identified as racial minorities, comparable to the demographics of the broader field (Butler et al., 2009). 
The participants recruited offer a distinctive sample of plastic surgeons that are particularly indicative of relations between consumerism and physician expertise. First, they were based in large, established cosmetic surgery markets in major metropolitan areas with welldeveloped patient word-of-mouth networks (e.g., Upper East Side of New York City). Second, because of the reliance on professional society membership lists for recruitment, interviewees have undergone the longest and most extensive available training for cosmetic surgery.

\section{Online reviews}

For all interview respondents, I collected star rankings posted on the review platforms RealSelf $(n=1,004)$ and Yelp $(n=404)$ on or before April 1, 2016 (see Table 1). I analyzed the content of 130 RealSelf reviews linked to ten randomly selected respondents. While other review platforms exist, I selected Yelp and RealSelf because surgeons mentioned them most often as sites they knew. RealSelf, a privately held company modeled after the travel review platform TripAdvisor, is the largest review platform for cosmetic procedures in the U.S. Yelp, a publicly traded company, encourages reviews on an array of services, with medical reviews constituting a small segment of overall offerings. On both sites, surgeons may register to "claim" their profiles for free and/or advertise their practices. Fake or defamatory reviews violating each site's Terms of Service can be removed by site staff.

On RealSelf, patients can write reviews, post photographs and ask questions on discussion boards to be answered by registered physicians. The review template asks patients to evaluate physicians on a scale of 1 to 5 stars on features such as bedside manner, follow-up, answering questions, staff professionalism, and phone/email responsiveness, which are all averaged into an overall star rating. In addition to giving an overall designation of "Worth It," "Not Sure," or "Not Worth It" for a procedure, reviewers comment narratively over time about 
issues ranging from selecting a doctor all the way through recovery. Each post by a consumer for a given business/physician constitutes one review in this study.

Though information on the identity of cosmetic surgery reviewers is proprietary and of limited availability, reviewers might be expected to be younger, more technologically savvy, and enjoying greater access to the internet (Hargittai and Walejko, 2008). Most U.S. cosmetic patients are white women, ranging from middle to upper class, though cost has become less of a barrier to cosmetic patients due to financing availability (Haiken, 1997). Reviewers are likely to have more strongly positive or negative experiences than the average patient. However, I am interested in surgeons' perceptions of these outliers, regardless of their representativeness. Analysis

Transcripts and reviews were initially coded and analyzed thematically using a constructivist grounded theory approach (Charmaz, 2001). In interviews, surgeons were directly asked about their views on RealSelf, with follow-up probes about online health information sharing and trends over time in their practice. Codes for physician authority and expertise came from the extant literature. Codes for reputation, evaluation standards, technical abilities, aesthetic standards, and customer service evaluations emerged through iterative reading of the data and composition of analytic memos. In the constructivist grounded theory approach, data and analysis represent the shared experiences of researcher and participants, offering "an interpretive portrayal of the studied world" (Charmaz, 2001, 678). By comparing surgeon statements, observations, and the content of reviews, I ensured that my analytic framework was not overly determined by any single narrative.

\section{The Effect of Reviews on Reputation and Authority}


As in other analyses of online reviews (Adams, 2011; López et al., 2012; Khansa, Khansa \& Pearson, 2016), I find that reviews were positive, offering detailed advice for prospective patients. However, surgeons remained skeptical of reviews, discussing them primarily in terms of reputation management. Indeed, at the annual ASAPS meeting, ten different sessions on reviews focused on strategies for reputation management. The ASAPS exhibit hall boasted thirty-odd vendors offering reputation services such as enhanced surgeon profiles on review sites, review monitoring, and search engine optimization to ensure that surgeons appeared in the best possible light online. In what follows, I discuss the content of reviews and show how their reputational effects influenced the doctor-patient relationship.

\section{Content Analysis of Online Reviews}

In reviews, cosmetic surgery appeared less like medical care as traditionally conceived economically and more like a service. Accordingly, qualitative comments were pitched as advice to prospective patients, with patients highlighting their own lay, embodied expertise acquired through their experience with surgery. Following the templates provided by review platforms, patients focused criticism of and feedback to doctors on customer service elements rather than surgeon's technical ability, and reviews were largely positive. In reviews for cosmetic surgery, price was an important component of overall value (De Langhe et al., 2015).

In aggregate, Yelp and RealSelf reviews for interview respondents were notably positive (Table 1). The average star rating of interviewees on RealSelf was 4.84 (out of 5), and twentyfour of respondents received exclusively 5-star reviews. Surgeons' average star-rating on Yelp was only slightly lower at 4.41 stars (see Figure 1). Of the ten surgeons whose RealSelf reviews were analyzed qualitatively, $90 \%$ of reviews received "Worth It" ratings on RealSelf, with $8 \%$ of reviews saying "Unsure" and 2\% saying "Not Worth It" (the latter ratings spread out amongst six 
different surgeons). With such high average star and "Worth It" ratings, patients clearly did not only post reviews when disgruntled or dissatisfied.

In most reviews, patients hewed closely to the RealSelf template, focusing their evaluations on customer service and aesthetic results. For example, beginning a review entry with "I love my breasts and tummy!", a 34-year-old female abdominoplasty and breast augmentation patient LB touted her surgeon's "excellent bedside manner" and willingness "to work with you if minor adjustments are needed at no cost!" She called her surgeon "exceptional" as a plastic surgeon and "human being." By noting his willingness to "work with you" in giving free adjustments, she emphasized the ease of the interaction and his pleasant manner. However, despite giving her doctor a five-star rating and grading the procedures "Worth It," she expressed disappointment with some "minor things like scars and some fat around [her] hips that [she] hates" in a one-year follow up post. Though satisfied, she indicated that the aesthetic result could have been better without directly blaming the surgeon. The metric of "Worth It" on RealSelf positioned patients-reviewers as consumers, with an emphasis on getting one's money's worth. If the price was right, surgeries could be "Worth It" even if patients had lingering "minor things like scars." Thus, ratings of five stars and "Worth It" were accompanied by nuanced qualitative portraits of patients' experiences, detailing the ups and downs of the surgical process (Orlikowsi and Scott, 2014).

That so few patients rated surgeons as "Not Worth It" and "Not sure" indicated patients' reluctance to criticize their cosmetic surgeons even if they were not fully satisfied with their outcomes. Patients in this sample only faulted surgeons when they were deeply unhappy or surgical complications arose. Of the 130 RealSelf reviews analyzed qualitatively, there were three "Not Worth It" reviews, all criticizing both the surgeon's bedside manner and their 
aesthetic outcomes. These patients complained about the lack of time that their surgeons spent with them and the "careless job" the surgeons did, pointing to imperceptible changes or "mismatched" asymmetries. These reviews were brief. In a typical negative review, facelift patient NP protested that the doctor "spent less than 5 min on my second consultation... Not only I look wired [sic]," but it was difficult to talk "since my muscle was pulled back so much!" The patient censured the doctor for his limited attention and the aesthetics of the outcome. Instead of improving her appearance, the outcome was a dysfunctional hampering of speech, clearly constituting a surgical failure.

The critical exceptions suggested a general rule of patient deference to surgeons. Even after a botched surgery, patients, uncertain what to expect, accorded surgeons expertise over technical matters and confined their evaluation primarily to customer service and aesthetics. For instance, though a young female patient "noticed one side of [her] nose bridge is a bit higher than the other side" after rhinoplasty, she "just let it slide for many years," accepting her surgeon's assertion that "everything was going to be fine." Only after "many years," did she act on her own negative evaluation of the asymmetric aesthetic result by getting a second medical opinion from a new surgeon about revision rhinoplasty. Even as this patient criticized her previous surgeon, she upheld physician authority in drawing on other surgeon's assessment to bolster her own in her review (Fox, 2005).

Regardless of the categorical rating, narrative, detailed reviews served to prepare prospective patients for cosmetic surgery, offering advice based on patients' expertise as consumers and patients. Some reviewers related their conflicts and reconciliation with surgeons for the benefit of their peers. Breast augmentation patient SB wrote, contra her surgeon, "my advice is to go bigger and not fear round breasts!! I pushed my way to $425 \mathrm{cc} \ldots$ \& even wish I'd 
been allowed go a little bigger!" Less than a month later, she followed up, "I wish this site let you edit previous posts...I'm a lot happier with my results now. I thought my constraints were aesthetic but after having a sit down with my provider, I feel a lot more informed.” As with SB, most patients clearly targeted their comments toward prospective patients, as indicated by the use of the imperative mode and the types of detail provided. A review from a breast augmentation patient concluded: "Be sure to take your meds on time, find what works for you, be prepared to sleep uncomfortably (maybe?), and work through the constipation." Such detail served to assert patients' hard-won knowledge based on their surgical experiences.

Thus, reviews were mostly positive and nuanced (Adams, 2011). Describing their experiences as a guide for future patients, patients largely offered satisfied accounts that justified their use of cosmetic surgery, showing formerly unhappy patients made happier by surgery (Haiken 1997). The lack of negative reviews suggests that bad reviews not only implicated surgeons as bad or incompetent but also implied a bad patient, someone whose problems could not be solved by cosmetic surgery. Ultimately, review platforms constituted a community of online users (Foster, 2016), with some reviewers offering year-long journals reflecting their feelings at multiple moments. In reviews, patients continued to defer to physician authority even as they asserted their embodied expertise.

\section{Cosmetic surgeons' perceptions of online reviews}

However, there was a gap between the actual content of reviews and what surgeons expected the content of reviews to be. Cosmetic surgeons' accounts of reviews emphasized their negative potential effects on their reputations. Adopting an active, defensive stance toward reviews, an article in the Aesthetic Surgery Journal urged surgeons to resist "let[ting] competitors, disgruntled employees, and unhappy patients manage [their] reputation, and watch 
[their] business decline" (Escoffery and Bauer, 2012, 652). Most surgeons contested the authenticity and commerciality of reviews, decrying the threat—and ignoring the boost—reviews posed to their reputations.

\section{Authenticity}

Most surgeons questioned the authenticity of reviews. Indeed, the first question asked by the audience at an ASAPS panel on reviews including the CEO of RealSelf was, "What percentage of the reviews on your website are fake?" (ASAPS Fieldnotes 4/7/2016). Several surgeons suggested that reviews could be manipulated. Some pointed out that positive reviews could be faked by unscrupulous competitors, and that negative reviews could be removed by platforms to promote advertising surgeons' interests. Respondents worried that this was more likely to happen on platforms like RealSelf and Yelp that mixed marketing and consumergenerated content. Moreover, surgeons expected negative reviews to be over-represented. As Dr. Murta explained, "If you're pissed off, then you Yelp for sure, because that's your way of lashing out. So Yelp has capitalized on that.” Dr. Murta suggested that review platforms took advantage of angry patients, providing them with a platform for smearing surgeons' reputations publicly. Called the "National Enquirer of the internet" by a surgeon presenting at the ASAPS conference, Yelp attracted particular ire from cosmetic surgeons for the perceived ease of manipulation of reviews on the site. According to these surgeons, reviews were less "authentic" and more "calculated" than they appeared (Jeacle and Carter, 2011; Lupton, 2014).

However, some surgeons accepted reviews as authentic portrayals of patient experiences. Reviews derived authenticity from the aggregation of star ratings and the personal quality of patients' testimony (Orlikowski and Scott, 2014; Adams, 2011). As Dr. Mannheim remarked, "People rely on reviews for restaurants, for mechanics... and doctors, in many ways, are no 
different because not everybody knows somebody who's had a facelift that they can trust to ask." In his view, reviews provide an authentic word-of-mouth referral. Likening the doctor-patient relationship to the often fraught mechanic-customer relationship, Dr. Mannheim took for granted that the customer would require outside assurances about the provider's ability, not trusting surgeon's professional norms as safeguards. Online reviews expanded the pool of trustworthy individuals to consult about surgical experiences (Brady et al., 2016), with third-party platforms guaranteeing the integrity and authenticity of the reviews (Jeacle and Carter, 2011). For these surgeons, reviews were free, authentic patient testimony on their behalf, building their reputations. Some surgeons believed in the authenticity of reviews precisely because patients were also "putting their bad results on the internet" (Dr. Kozlowski), giving patients a unique and seemingly unvarnished depiction of surgery. Dr. June offered measured praise of RealSelf as "reinforc[ing] what I'm saying sometimes, that this is normal swelling, discomfort, blah, blah, blah. Patients are like, 'Oh okay, I read that, and I've seen that before, pretty consistent'." In this view, reviews could bolster physician authority, boosting their reputation and confirming their expertise by providing an additional independent source of counsel usually in agreement with surgeons' advice (Song et al., 2012).

\section{Commercial interests}

Some critical surgeons asserted that review platforms had an interest in maintaining negative reviews whether or not they were authentic, which was also not in patients' best interest (Jeacle and Carter, 2011). Surgeons accused third-party platforms of being tainted by commercial motives. As Dr. White expressed with some frustration, "It's driven by the people that own the site, money for them." Surgeons like Dr. Murta thought that reviews for cosmetic surgery were more likely to be negative because the anonymity of online reviews allowed 
unhappy patients to complain about their surgeons without facing the social stigma associated with undergoing cosmetic surgery. Despite studies showing that online reviews are generally positive (López et al., 2012; Khansa, Khansa \& Pearson, 2016), many respondents were convinced that review platforms did not advance their financial or professional interests, seeing platforms as facilitating reputational damage on an unprecedented scale.

To combat surgeons' antipathy toward reviews and appeal to surgeons' financial interests, RealSelf and others offered differing services for reputation management. RealSelf provided report cards for surgeons to see how their online metrics compared with their regional competitors; reports from user survey data; enhanced profiles; and high-traffic discussion boards (ASAPS fieldnotes, 4/7/2016). In offering such services for surgeons in addition to online reviews for patients, RealSelf staff described these efforts as helping surgeons help "consumers to overcome the information gap, and the asymmetry they feel in their position" (ASAPS Fieldnotes, 4/7/2016). But many respondents preferred to engage other companies to monitor their reviews and alert them when they "needed to correct" a bad review (Dr. Johnson). "Correction" included asking platforms to remove defamatory reviews and checking in with unhappy patients. Several online marketing firms advertised reputation management services at the annual ASAPS meeting, epitomized by the following typical tagline from Doctor.com:

When people look for you or your practice, are they finding the real you, or are they only finding one or two unrepresentative negative reviews? Take control of your reputation with a fast, foolproof way to encourage your current satisfied patients to speak up (ASAPS Fieldnotes, 4/5/2016).

Reputation management services promised surgeons more control, capitalizing on surgeons’ fears of "one or two unrepresentative negative reviews" to garner subscriptions. 
Other surgeons rejected paid efforts to manage their online reputations as the latest front in marketing. Dr. Allison remarked, "It's just a little bit selling out to do that... some people are really good at that. I'm not. I'm not really good at marketing." Here she equated responding to review platforms with marketing, which was also suspect in her mind. According to this logic, surgeons earned their reputations through their surgical outcomes and need not solicit patient testimony or feedback. Surgeons wanted their reputation to stem from their expertise and professionalism, not from patient opinion in the form of reviews, and they resented what they saw as encroachment on their turf.

Effect of online reviews on the doctor-patient relationship

Cosmetic surgeons felt that reviews turned the balance of power against them in the doctor-patient relationship, facing increased scrutiny from patients. In interviews, surgeons reported changing their behavior, or reactivity to, reviews; that is, surgeons acted differently to garner better reviews (Espeland and Sauder, 2016). As a result, some surgeons felt that they were being monitored by an invisible, online audience. Dr. Solomon described a change in the doctorpatient relationship: "The dynamics between the patient and doctor have moved outside that oneto-one dynamic. It's become more of a social issue. Where before, breast augmentation was an issue between women, maybe her spouse, her friends, and her doctor." Surgeons interpreted online reviews as indicators of heightened scrutiny by patients, congruent with Conrad et al.'s (2016, p.22) observation of "the transformation of illness from a largely private to an increasingly public experience" due to the internet. Discussed as sousveillance by other health information scholars (Adams, 2014), this "watching from below" of doctors by their patients gave patients more power in an unequal relationship. But many surgeons were cynical about the 
extent to which this shift empowered patients. In Dr. Solomon's reasoning, the invisible online audience made social norms even more of a profound influence on patients' behavior.

Though attentive to reviews in the aggregate, surgeons did not cite particular reviews as motivating changes they made in their practice, consistent with other scholarship on reviews as a form of post-panoptic surveillance (Adams, 2014). They did, however, anticipate patients' review responses when making recommendations that might upset patients. Known for their bluntness, surgeons reported toning down their language or censoring themselves for fear of their potential effect on their reviews. For instance, to avoid offense, surgeons reported being reluctant to tell patients that their body mass index (BMI) was too high for safe use of liposuction. Whereas before they would have counseled patients to lose weight and return with a lower BMI, surgeons now simply said liposuction was not an option for the patient, sidestepping an uncomfortable topic. Some surgeons were reluctant to say no to patients outright, no matter how impossible a patient's request, for fear of how they might be reviewed. In describing this heightened awareness of how they could be evaluated by patients, Dr. Kozlowski observed,

Doctors are almost afraid now not to give someone narcotics who's drug-seeking because they'll get a bad rating on somebody's dotcom. So these ratings [patients] look at that and they look for the doctors with the most gold stars. It doesn't mean they're any good.

Dr. Kozlowski emphasized that surgeons feel pressured to change their behavior to garner better reviews, such that reviews were therefore not a good indicator of surgeons' ability. Since, unlike good surgical results, good reviews had "nothing to do with your skills, credibility" (Dr. Hanson), some surgeons ignored them. Despite injunctions from ASAPS staff that "it takes more than expertise in the operating room to produce a happy patient" (Nahai, 2014, 190), most 
surgeons dismissed reviews as unfair evaluations of their surgical skill, ignoring them as actionable feedback on customer service. Thus, the proliferation of online reviews and patients' use of them did not necessarily translate to better patient outcomes.

From how surgeons discussed reviews, it is clear that cosmetic surgeons did not see patients as qualified to comment on their work (Adams, 2011). Surgeons saw reviews as, in the best case, complementing their authority and boosting their reputation as free marketing. In the worst case, they portrayed reviews as unfairly maligning their reputations. With careful management, as with judicious advertising, surgeons felt reviews could benefit their practice. Cosmetic surgeons modified their behavior to avoid negative reviews, but in an attempt to manage their reputation rather than to improve their practice. And a minority of cosmetic surgeons interviewed reported a discrepancy between the kind of behavior that they knew would garner them better reviews and what surgeons saw as the best professional course of action.

\section{Discussion}

\section{Authority and the doctor-patient relationship}

Online reviews make visible two components of physician authority: expertise (knowledge and skills), of primary importance, and secondarily, reputation (the social reception and perception of surgeons' ability and expertise). Expertise and reputation are related. Reputation is in part a function of surgeon's expertise; an unskilled surgeon prone to complications will likely develop a bad reputation. However, physician reputation also incorporates patients' subjective experiences of service, their perception of a given physician relative to others, and other reviews, such that a good reputation does not necessarily signify an exceptionally technically-expert surgeon. Conversely, a bad reputation can cast aspersions on 
surgeons' expertise amongst prospective patients, even if a surgeon has excellent credentials and peer recognition.

Reviews also change the doctor-patient relationship. Scholars have posited the doctorpatient relationship as a power dynamic in which physicians have authority over patients stemming from their specialized knowledge, sometimes conceptualized as expertise (Starr, 1982). In this model, patients lack the knowledge to evaluate their physicians' expertise or to treat themselves, relying on the medical profession for both evaluation and treatment. In reviewing physicians, patients assert a lay, embodied expertise rooted primarily in their roles as consumers, evaluating their experience as they would other services. This embodied/lay expertise as consumers gives patients the power to shape surgeons' reputations by expressing their satisfaction (or lack thereof) with their surgical results. By publishing their discontent, even as guidance, for a broader online community, patients can threaten surgeons' reputations and thereby their authority. Ultimately, patients claim lay expertise primarily over service and their subjective experience. Reviews have changed the doctor-patient relationship by prompting surgeons to treat patients with more courtesy (e.g., toning down their language), but the symbolic boundary between surgeons' objective medical expertise and patients' subjective lay experience remains.

Therefore, authority does not necessarily accrue to individual patients Reviews derive their potential impact from being seen by a larger public, and access to that larger public is moderated and mediated by the companies hosting online review platforms (Adams, 2014). Review platforms aim to reduce the information asymmetry between patients and doctors, chipping away at patients' singular dependence on one physician. Due to the large base of 
potential patients and users they can reach, the very existence of review platforms challenges physician authority, above and beyond any particular review.

Surgeons fear patients' power as consumers, as well as the review platforms' power as mediators of their relationship with patients. They therefore dismiss reviews pre-emptively. Though patients elect whether or not to post reviews, surgeons are conscripted onto review platforms regardless of their consent. Their inability to resist this conscription partially undergirds their skepticism of reviews and especially review platforms, despite the friendly overtures that platforms have made toward them.

\section{Review positivity}

Most cosmetic surgeons ignore the fact the reviews have positive as well as negative reputational effects. For my respondents, average star ratings are generally high, especially on RealSelf, with positive reviews bolstering physician reputation and authority. In fact, reputations are only under threat from review platforms because platforms augmented and elevated surgeons' reputations in the first place. Some surgeons recognized this positive effect, enrolling their satisfied patients to post on their behalf. Review platforms recognized it, dismissing surgeons' complaints as failing to see their potential gain. And ASAPS leadership and staff recognized it, attempting to broker better relations between review platforms and surgeons. Amongst my interview respondents, the positive reputational effects of reviews are overshadowed by the rare horror stories of bad reviews.

Some surgeons saw online reviews and the scrutiny they represented as diminishing their authority, with their reputations hanging in the balance. Authority devolved primarily to review platforms and the wider invisible audience of the internet (Adams 2014). Review platforms treated cosmetic surgery seekers as customers rather than patients, altering the dependence of 
patients in the doctor-patient relationship. The evidence presented here suggests that contemporary physicians' conceptualization of patients as consumers is circumstantial and contingent, exacerbated by the advent of technological platforms that, in surgeons' minds, increase the distance between surgeons and patients by introducing other parties into the doctorpatient relationship.

\section{Reach of online reviews in medicine}

As reviews become a bigger feature of the medical landscape, it is worth speculating on the extent of their reach. Certainly, physicians assigned to patients because of their proximity and/or based on insurance coverage are more insulated from reviews; in contexts with minimal choice, patients may be unhappy with their providers, but may be stuck with them if they want to receive treatment. Reputation becomes a more salient aspect of physician authority in medical contexts in which patients have a choice of provider. I anticipate reviews to have similar effects on physician authority and the doctor-patient relationship for certain cash pay medical professionals, such as ophthalmologists, dentists, dermatologists, ob-gyns, and/or complementary and alternative medicine practitioners, who are less dependent on insurance payments due to specialization in particular elective procedures.

Though cosmetic surgery is an exemplary case for highlighting the potential importance of physician reputation, surgeons are differentially subject to the effects of reviews. Reviews do not singlehandedly determine reputation: Most cosmetic surgeons, especially long-time practitioners, depended on word-of-mouth referrals from patients, with recruitment occurring offline in existing social networks (e.g., the Upper East Side of New York City). In these cases, though patients may still consult reviews before committing to a surgeon, the testimony of a family member or friend counted for more, insulating these surgeons from the consequences of 
negative reviews. For surgeons practicing in less-dense areas or those just starting out, reviews could likely do more to hurt their reputations and dissuade prospective patients.

In contrast to existing scholarship (Adams 2011; Adams 2014), I thus situate cosmetic surgery review platforms as outside the health institution mainstream and therefore more of a threat to physician authority. Cosmetic surgery in the U.S. is private in every sense: no public funds can be used for it, private health insurance does not generally cover it, and cosmetic surgeons are not employed by the government. Unlike other platforms owned by surgeons or medical commercial interests, RealSelf and Yelp are private commercial platforms outside of the institutional apparatus of doctors, professional associations, clinics, and regulatory agencies. Lacking such connections, these platforms can position themselves as putting patient-reviewers first, whether they do or not. The case of cosmetic surgery thus suggests what can and does happen when the state plays a backseat role in medical provision and evaluation.

\section{Conclusion}

To understand the effect of online reviews on the doctor-patient relationship, this paper considers two contributors to physician authority: reputation and expertise. Cosmetic surgeons experienced negative reviews as a challenge to physician authority because of their adverse reputational effects. But positive reviews, which predominated, bolstered cosmetic surgeons' authority by reinforcing physician expertise and enhancing physician reputation. Review platforms offered patients an opportunity to exchange information about cosmetic surgery in lay terms as consumers, articulating their own embodied expertise (Epstein, 1995; Prior, 2003). Patients also exhibited expertise as consumers, evaluating their surgeons on a limited set of characteristics in line with the constraints of review platforms (Lupton, 1997). Qualified by their status as consumers, patients emphasized surgeons' customer service abilities over surgeons' 
professional credentials and abilities. As they wrote and consulted online reviews, patients inhabited both the "reflexive subject" and "dependent patient" modes while continuing to accord significant authority to their chosen cosmetic surgeon (Henwood et al., 2003; Lupton, 1997).

An analysis of reviews alone would suggest that online reviews complement rather than challenge physician authority because of the deference patients accorded to physician expertise in reviews. However, interviews with surgeons made clear that surgeons perceived reviews as a challenge to their authority by providing patients with the opportunity to undermine their reputations. Accordingly, surgeons responded to the potential of negative reviews rather than the reality of nuanced or positive reviews. Moreover, surgeons feared the power of the third-party companies hosting reviews. Surgeons described modifying their practice to avoid negative reviews by offering good customer service, recruiting happy patients to post on their behalf, and avoiding touchy subjects in discussions with patients.

Online reviews are but one information source in a larger information ecology. Relatively novel phenomena, they are influential especially at the local level, providing a personal authenticity that "expert," objective guides cannot. Information asymmetry between providers and patients characterizes medical interactions, as physicians possess specialized technical knowledge that is difficult for patients to evaluate. My findings suggest that in consumer-driven medical contexts in which patients have a choice in their healthcare providers and a public platform on which to review their experience, physicians may feel a loss of authority stemming from the vulnerability of their reputations.

\section{References}

Adams, S.A. (2011). Sourcing the crowd for health services improvement: The reflexive patient and 'share-your-experience' websites. Social Science \& Medicine, 72, 1069-1076. 
Adams, S. (2013). Post-panoptic surveillance through healthcare rating sites. Information, Communication \& Society, 16, 215-235.

Barker, K.K. (2008). Electronic support groups, patient-consumers, and medicalization: The case of contested illness." Journal of Health and Social Behavior, 49, 20-36.

Brady, E., Segar, J., \& Sanders, C. (2016). 'You get to know the people and whether they're talking sense or not': Negotiating trust on health-related forums. Social Science \& Medicine, 162, 151-157.

Broom, A. (2005). Virtually he@lthy: The impact of internet use on disease experience and the doctor-patient relationship. Qualitative Health Research, 15(3), 325-45. doi:10.1177/1049732304272916.

Butler, P., Britt, L.D., \& M.T. Longaker. (2009). Ethnic diversity remains scarce in academic plastic and reconstructive surgery. Plastic Reconstructive Surgery, 123, 1618-1627.

Charmaz, K. (2001). Qualitative interviewing and grounded theory analysis. In J.F. Gubrium \& J.A. Holstein (Eds.), Handbook of interview research (pp. 675-694). Thousand Oaks, CA: Sage Publications.

Chiu, Y. (2011). Probing, impelling, but not offending doctors: The role of the internet as an information source for patients' interactions with doctors. Qualitative Health Research. 21, 12, 1658-66. doi:10.1177/1049732311417455.

Clarke, A., Shim, J.K., Mamo, L., Fosket, J.R., \& Fishman, J.R. (2003). Biomedicalization: Technoscientific transformation of health, illness, and U.S. biomedicine. American Sociological Review, 68(2), 161-194.

Conrad, P., Bandini, J. \& Vasquez, A. (2016). Illness and the internet: From private to public experience. Health: An interdisciplinary journal for the social study of health, illness and 
medicine, 20(1), 22-32.

De Langhe, B., Fernbach, P.M., \& Lichtenstein, D.R. (2015). "Navigating by the stars: Investigating the actual and perceived validity of online user ratings." Journal of Consumer Research, 42, 817-833.

Epstein, S. (1995). The construction of lay expertise: AIDS activism and the forging of credibility in the reform of clinical trials. Science, Technology \& Human Values, 20(4), $408-37$.

Espeland, W. \& M. Sauder. (2016). Engines of anxiety: Academic rankings, reputation, and accountability. New York: Russell Sage Foundation.

Eyal, G. (2013). For a sociology of expertise: The social origins of the autism epidemic. American Journal of Sociology, 118(4), 863-907.

Fine, G.A. \& Xu, B. (2012). Honest brokers: The politics of expertise in the 'Who Lost China?' debate. In G.A. Fine (Ed.), Sticky reputations: The politics of collective memory in midcentury America (pp. 151-182). New York: Routledge.

Fox, N.J. \& Ward, K. J. (2005). The 'Expert Patient': Empowerment or Medical Dominance? The Case of Weight Loss, Pharmaceutical Drugs and the Internet. Social Science \& Medicine 60(6), 1299-1309. doi:10.1016/j.socscimed.2004.07.005.

Foster, Drew. (2016). 'Keep complaining til someone listens': exchanges of tacit healthcare knowledge in online illness communities. Social Science \& Medicine 166, 25-32.

Haiken, E. (1997). Venus envy: A history of cosmetic surgery. Baltimore: The Johns Hopkins University Press.

Hargittai, E. \& Walejko, G. (2008). The participation divide: Content and creation and sharing in the digital age. Information, Communication \& Society, 11(2), 239-256. 
Henwood, F., Wyatt, S., Hart, A., and J. Smith. (2003). 'Ignorance Is Bliss Sometimes': Constraints on the emergence of the 'informed patient' in the changing landscapes of health information. Sociology of Health \& Illness, 25(6), 589-607.

Jeacle, I. \& Carter, C. (2011). In TripAdvisor we trust: Rankings, calculative regimes and abstract systems. Accounting, Organizations and Society, 36, 293-309.

Khansa, I., Khansa, L., \& Pearson, G.D. (2016). Patient satisfaction after rhinoplasty: A social media analysis. Aesthetic Surgery Journal, 36(1), 1-5.

Lamont, M. (2012). Toward a comparative sociology of valuation and evaluation." Annual Review of Sociology, 38, 21.1-21.21.

López, A., Detz, A., Ratanawongsa, N., \& Sarkar, U. (2012). What Patients Say about Their Doctors Online: A Qualitative Content Analysis.” Journal of General Internal Medicine 27 (6): 685-92.

Lupton, D. (2014). The commodification of patient opinion: The digital patient experience economy in the age of big data. Sociology of Health and Illness, 36(6), 856-869.

Lupton, D. (1997). Consumerism, reflexivity and the medical encounter. Social Science \& Medicine, 45(3), 373-81.

Mueller, M.A., Glassett, T.S., Burke, S.M., \& Nichter, L.S. (2013). Protecting a plastic surgeon’s reputation: Damage control for dishonest online reviews. Plastic and Reconstructive Surgery Journal, 131(4), 670e-671e.

Nahai, F. (2013). “Commercialization: Defining our terms.” Aesthetic Surgery Journal, 33(7), 1069-1070.

Orlikowski, W.J. \& Scott, S.V. (2014). What happens when evaluation goes online? Exploring apparatuses of valuation in the travel sector. Organization Science, 25(3), 8688-891. 
Patel, S., Cain, R., Neailey, K., \& Hooberman, L. (2015). General practitioners' concerns about online patient feedback: Findings from a descriptive exploratory qualitative research in England. Journal of Medical Internet Research, 17(12), e276.

Porter, T. (1995). Trust in numbers: The pursuit of objectivity in science and public life. Princeton: Princeton University Press.

Prior, L. (2003). Belief, knowledge and expertise: The emergence of the lay expert in medical sociology. Sociology of Health \& Illness, 25(3), 41-57.

Song, F.W., West, J.E., Lundy, L., \& Dahmen, N.S. (2012). Women, pregnancy, and health information online: The making of informed patients and ideal mothers. Gender \& Society, 26(5), 773-98.

Starr, P. (1982). The social transformation of American medicine: The rise of a sovereign profession and the making of a vast industry. New York: Basic Books.

Sullivan, D. (2000). Cosmetic surgery: The cutting edge of commercial medicine in America. New Brunswick: Rutgers University Press. 
Table 1.

\begin{tabular}{|l|l|l|l|l|}
\hline $\begin{array}{l}\text { Online } \\
\text { Review } \\
\text { Platform }\end{array}$ & $\begin{array}{l}\text { Number of } \\
\text { interviewed doctors } \\
\text { with reviews on this } \\
\text { platform }\end{array}$ & $\begin{array}{l}\text { Number of } \\
\text { reviews total in } \\
\text { the sample }\end{array}$ & $\begin{array}{l}\text { Average } \\
\text { star rating } \\
\text { (out of 5) }\end{array}$ & $\begin{array}{l}\text { Standard } \\
\text { deviation in } \\
\text { star rating }\end{array}$ \\
\hline RealSelf & 31 & 1004 & 4.84 & 0.30 \\
\hline Yelp & 28 & 404 & 4.41 & 0.77 \\
\hline
\end{tabular}

Table 1. Descriptive statistics of star ratings for interview participants by online review platform. 
Figure 1.

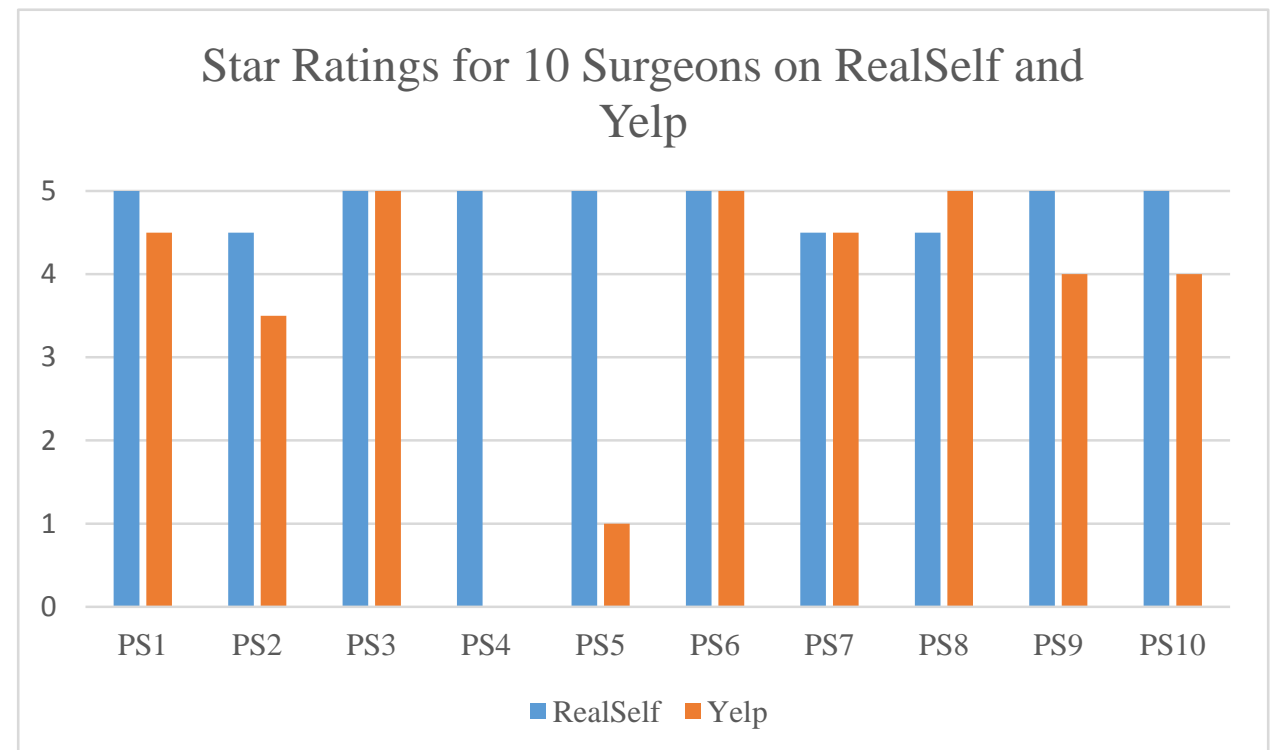

Figure 1. Ratings are presented out of five stars. " 1 " is the worst rating, " 5 " is the best. PS4 and PS5 had no posted Yelp reviews. 